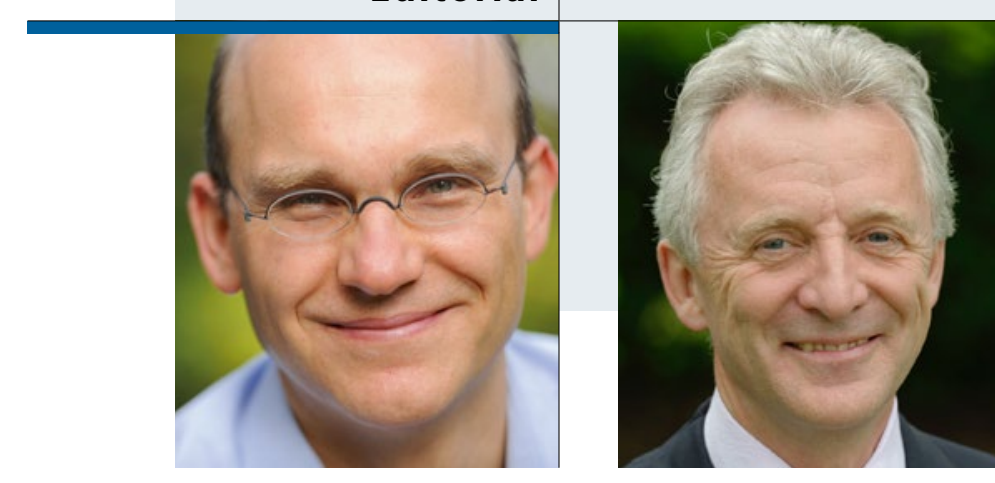

"Wir hoffen, Ihnen einen Einblick in ein

faszinierendes und vielfältiges Fachgebiet

zu geben."

Dr. Stephan Buderus, 1. Vorsitzender der GPGE,

St.-Marien-Hospital, Bonn

Dr. Martin Claßen, 2. Vorsitzender der GPGE,

Klinikum Links der Weser, Bremen

\title{
Pädiatrische Gastroenterologie, Hepatologie und Ernährung
}

D er Verdauungstrakt spielt mit seinen vielfältigen Funktionen und seinen Assoziationen mit anderen Organen eine zentrale Rolle für Gesundheit, Wohlbefinden und Lebensqualität. Der Gastrointestinaltrakt stellt die größte Oberfläche zur Umwelt dar, beherbergt die meisten immunkompetenten Zellen und spielt eine wichtige Rolle bei der Entwicklung von Toleranz und Immunität. Gerade bei Kindern ist eine ausreichende Aufnahme von Nährstoffen entscheidend für eine optimale körperliche und geistige Entwicklung. Dass das intestinale Mikrobiom und seine Wechselwirkungen im Gastrointestinaltrakt einen bunten Strauß von Erkrankungen wie zum Beispiel Adipositas, Diabetes mellitus oder Depression beeinflusst, revolutioniert unser Gesundheits- und Krankheitsverständnis.

Die Besiedlung des kindlichen Gastrointestinaltraktes mit Bakterien erfolgt in den ersten Lebenstagen und wird unter anderem auch durch ärztliche Maßnahmen erheblich beeinflusst. Deshalb tragen wir als Pädiater eine besondere Verantwortung - sowohl ganz grundlegend in Hinblick auf Aufklärung und Beratung der Familien als auch in Bezug auf die Bedeutung und möglichen Konsequenzen einer medizinischen Therapie.

Anlässlich der gemeinsamen Jahrestagung der Gesellschaft für Pädiatrische Gastroenterologie und Ernährung (GPGE) mit der DGKJ vom 14.-17. September 2016 in Hamburg haben wir Ihnen in dieser Sonderausgabe aktuelle und praktisch wichtige Themen aus der pädiatrischen Gastroenterologie, der Hepatologie und der Ernährungsmedizin zusammengestellt.

Eine häufige, aber vermutlich immer noch unterdiagnostizierte Erkrankung ist die Zöliakie. Aus diesem Grunde gehören die aktuellen Ergebnisse zur Prävention und die Empfehlungen zur Diagnose und Therapie ganz zentral in ein pädiatrisch-gastroenterologisches Sonderheft. In einer Kasuistik wird ein besonderer Fall einer Zöliakie vorgestellt. Wie die Lebensqualität von CED-Patienten verbessert werden kann, ist ebenfalls Thema dieses Supplements. Ein nächster Artikel fokussiert auf eine deutlich kleinere Patientengruppe, die aber - nicht zuletzt deshalb - besonderer Aufmerksamkeit und hochspezialisierter Versorgung bedarf: Es geht um Ursachen, Diagnostik und Therapie des chronischen Darmversagens.

Der nächste Themenblock betrifft die Leber. Ein Ikterus beim Neugeborenen und jungen Säugling ist nicht immer harmlos. Vielmehr erfordert eine neonatale Cholestase eine frühzeitige und spezifische Abklärung, damit gegebenenfalls notwendige therapeutische Schritte schnell eingeleitet werden können. Leberwerterhöhungen bei pädiatrischen Patienten jeden Alters sollten auch an infektiöse Hepatitiden denken lassen. Ein Artikel präsentiert ein Update zur Therapie.

Die sogenannte FODMAP-arme Kost ist eine derzeit viel diskutierte neue Kostform, deren Einsatz bei verschiedenen gastrointestinalen Beschwerden zum Teil unkritisch empfohlen wird. In dem Beitrag wird eine aktuelle Bewertung vorgenommen. Beim Thema Malnutrition bei chronisch kranken Kindern geht es darum, aufzuzeigen, dass es auch bei uns im wohlversorgten Mitteleuropa manifeste Mangelernährung gibt.

Während unserer Tagung in Hamburg werden die Interaktionen mit anderen Organsystemen und die interdisziplinäre Kooperation von pädiatrischer Gastroenterologie mit anderen Organdisziplinen besondere Schwerpunkte auch fachübergreifender Sitzungen sein. Wir laden alle Interessierten ein, das wissenschaftliche Programm der GPGE zu besuchen. Wir hoffen, sowohl mit den Artikeln des Sonderheftes als auch mit den Präsentationen, Postern und Vorträgen der Tagung Ihr Interesse zu wecken und Ihnen einen Einblick in ein faszinierendes und vielfältiges Fachgebiet zu geben.

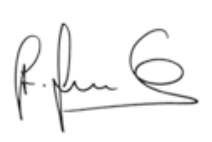

Dr. Stephan Buderus

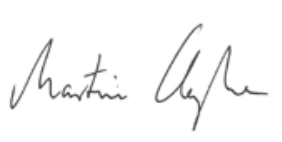

Dr. Martin Claßen 